\title{
WHY WOMEN CRY
}

What is the function of tears? A paper published in Science by a group from the Weissmann Institute of Science, Rehovot, Israel, describes that sniffing tears from women reduced sexual arousal in men (6 Jan 2011; doi:10.1126/science.1198331).

The men looked at pictures of women's faces while sniffing tears or saline. "To our surprise, we found no change in the area of positive or negative mood, but there was a decrease in sexual arousal," says Noam Sobel, the main author on the paper (New Scientist, 6 Jan 2011). Both self-reported and physiological measures of sexual arousal decreased, including a drop in salivary testosterone levels. Moreover, functional MRI showed reduced activity in the brain areas associated with sexual arousal, such as the hypothalamus.

"It's probably the best study yet showing that chemical communication between humans is a reality," says Thomas Cleland of Cornell University, Ithaca, New York, USA (National Public Radio, 7 Jan 2011). "This experiment opened gazillions of questions," says Sobel (Washington Post, 7 Jan 2011), including whether the chemical signal is a pheromone.

As for the function of this chemosignalling, Sobel says that "women cry much more during menstruation, which indeed from an evolutionary standpoint is not a beneficial time for sexual interaction" (Time Magazine, 6 Jan 2011).

The subjects responded to the tears without actually seeing the women cry. "We hug a crying loved one, often placing our nose near teary cheeks, typically generating a pronounced nasal inhalation as we embrace," says Sobel (Independent, 7 Jan 2011). However, according to Thomas Mast of Florida State University in Tallahassee, USA, "the visual impact of seeing someone crying is going to wipe over whatever chemical effects are found in tears" (USA Today, 6 Jan 2011). 